\title{
Parallel processing of position and form information in young children
}

\author{
JOANNA BLAKE \\ York University, Toronto, Ontario, Canada
}

\begin{abstract}
Young children and adults were compared in their processing of position and form information when only one type of information was processed and when both types of information were processed. For adults, position information in both the single and combined tasks showed a linear increase in report accuracy with increasing duration. For children, this linear increase was found only in the single task, at longer durations, resulting in a loss of parity across tasks for position information among children. No loss of parity was found for form information for either age group. The children's loss of parity in processing position information when combined with form information replicates their previously demonstrated inability to process multiple-form arrays using a parallel independent strategy.
\end{abstract}

The ability to process multiple-stimulus arrays in a parallel independent fashion increases with age (Blake, 1974). Preschool children showed some parallel independent processing as array size increased from one to two forms but did not maintain this strategy with larger array sizes, whereas older children and adults did.

The ability to process different types of information in parallel may also increase with age. Finkel (1973) found that the recall of position information by preschool children nearly equaled that of adults but abruptly dropped when one position marker was replaced by a form. Adults, on the other hand, seemed able to process position and form information in parallel and had difficulty only when the number of forms to be recalled began to exceed their processing limit. Finkel suggests that the processing systems for identity and spatial information in young children are separate and only gradually become integrated with development. However, separate mechanisms may still be operative in adults when the task is made more difficult by limiting processing time with a visual noise field (Smythe \& Finkel, 1974). Under these conditions, adults showed the same decrement as young children did in the previous study when required to report both position and form information. In addition, their rate of encoding position information was faster than their rate of encoding form information. This difference in encoding rate in favor of position information

This research was supported by Grant A8575 from the National Research Council of Canada. The assistance of Brian Heisel, Allen Walton, Sheilah McConnell, and Frank Reddy is greatly appreciated. I would also like to thank Professor Roger Schvaneveldt for his help with the trend analysis. Reprint requests should be sent to Joanna Blake, Department of Psychology, York University, 4700 Keele St., Toronto, Ontario, Canada. was also found by den Heyer (Note 1). Differential rates of decay for spatial as compared with identity information have been found in several studies using the partial report paradigm (Dick, 1969; Finkel \& Smythe, 1973; Turvey \& Kravetz, 1970). These differential rates of encoding and of decay have been interpreted as support for the notion that spatial and identity information are processed by separate mechanisms. Such a model positing separate systems can be contrasted with one in which spatial and identity information are processed as a unit (Cumming $\&$ Coltheart, 1969).

If spatial and identity information are, in fact, encoded by different systems, these systems may or may not operate independently of each other. The usual criterion for independence is performance parity, i.e., that "two perceptual processes are considered independent if performance on two perceptual tasks carried out simultaneously is the same as the sum of the performances on each task carried out separately" (Garner \& Morton, 1969, p. 235). However, performance on the single tasks cannot simply be compared with overall performance on the combined task because of increased error correlation in the latter. The proper comparison, according to Garner and Morton (1969), is between tasks in which a given type of information is reported when (1) only that information is presented, (2) more information is presented but not reported, and (3) more information is presented and reported. Comparison of performance with a given type of information across these three tasks allows a distinction between interference due to perceptual incapacity and interference due to response competition. den Heyer (Note 1), employing all three tasks, found a loss of parity across tasks, but the difference was in the intercept and not in the slope. He interprets the absence of variation in rate of encoding 
across tasks as parallel independent processing of spatial and identity information.

The present study was concerned with whether or not young children would also process these two types of information in a parallel independent fashion. Given the previous finding (Blake, 1974) that they have difficulty using this strategy with form information alone, it was expected that their ability to process two different types of information using a parallel independent strategy would be similarly limited. Like the studies of Smythe and Finkel (1974) and den Heyer (Note 1) with adults, a backward-masking paradigm was also employed in this study, based on evidence in favor of a stopprocessing or central view of backward-masking effects (Haber \& Standing, 1968; Liss, 1968). Of the tasks recommended by Garner and Morton (1969), only the first and third were administered because selective reporting of information is very difficult for young children under these conditions (Blake, 1974). Thus, parity was tested by comparing the processing of position and form information when each was presented alone vs. together, but loss of parity could not be attributed unequivocally to perceptual incapacity or response incompatibility. In accordance with den Heyer's (Note 1) results, no loss of parity was expected for adults, but children were expected to process position and form information faster when only one type of information was presented and reported than when both types of information were presented and reported.

\section{METHOD}

\section{Subjects}

The subjects were five children ranging in age from 5 years 7 months to 6 years 6 months, with a mean age of 5 years 11 months, and five York University students. No subject had had any previous tachistoscopic experience. The subjects were paid for their participation.

\section{Apparatus}

A standard Iconix four-field tachistoscope with associated control logic was used. The first three fields of the tachistoscope contained the fixation point, the stimulus array, and the masking array, respectively. The luminance of each field was approximately $51.88,49.33$, and $69.06 \mathrm{~cd} / \mathrm{m}^{2}$, respectively. The intervals between presentation of the fields were dark.

\section{Materials}

Examples of each of the three types of stimulus materials used are given in Figure 1.

$P$ cards. For the position information alone task ( $P$ task), the stimulus items were four $\times s$ which were assigned randomly to four of nine $1 / 2 \times 1 / 2$ in. cells of a $1 \frac{1 / 2}{2} \times 1 \frac{1 / 2}{\text { in. matrix, such }}$ that no pattern of four filled cells was repeated within a block of six trials and all cells were filled an equal number of times across all 72 trials on the $P$ task.

$F$ cards. For the form information alone task ( $F$ task), four different forms were assigned randomly to the four corners of a $1 \frac{1 / 2}{2} \times 1 \frac{1 / 2}{}$ in. square, such that each form appeared twice in two different locations within a block of six trials and an equal number of times in each location across all 72 trials on the $F$ task, and no combination of forms was repeated.
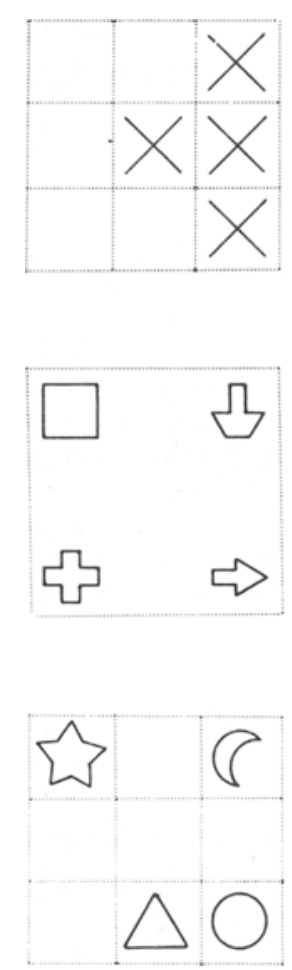

Figure 1. Examples of the stimulus cards used in the position information alone task ( $P$ task), the form information alone task (F task), and the position plus form information task (P $\times$ F task).

$P \times \mathbf{F}$ cards. For the position plus form information task $(P \times F$ task), the four forms were assigned randomly to four of the nine cells of the $1 \frac{1}{2} \times 1 \frac{1}{2}$ in. matrix, following the constraints for both sets given above.

The set of forms used was the same set of 12 forms illustrated in Blake (1974). The size of each form and $x$ was approximately $8 \mathrm{~mm}$, subtending $31^{\prime}$ of visual angle at the field-subject distance of $88.9 \mathrm{~cm}$. The masking stimulus for the $P$ cards contained an $x$ in each of the nine cells of the matrix. For the $P \times F$ cards, each cell was filled with a form that was a composite of four of the stimulus forms, the square, circle, arrow, and thin rectangle. For the $F$ cards, this composite form appeared at the four corners of the square.

$A$ response card, which was either a matrix (for the $P$ and $P \times F$ tasks) or a blank square (for the $F$ task), was mounted on a metal plate to the right of the T-scope viewing hood. Below it were either 4 magnets with $\times s$ on top (for the $P$ task) or 12 magnets with the 12 forms on top (for the $F$ and $P \times F$ tasks).

\section{Procedure}

Practice trials. Each of the three tasks, $P, F$, and $P \times F$, was introduced in turn with a practice trial without the $\mathrm{T}$-scope, followed by five T-scope trials at durations of 1,000 and $250 \mathrm{msec}$ without a mask and 250 msec with a mask. The subjects were told to make the response card look just like the stimulus card, except that on the F task they could put the pictures anywhere on the response card. Trials were self-paced; the subjects pushed a button to start each trial when they were looking into the $T$-scope and were ready. They were required to make four responses on each trial and encouraged to guess. The children were given checkers for correct responses, and these were exchanged for M\&Ms during breaks in the session. Adults were told how many of their four responses were correct on each trial.

Test trials. Test sessions were introduced by a fast series of descending and ascending trials with the mask so that the subjects 
would quickly become reaccustomed to the T-scope. For each task, a block of six trials was given at stimulus durations of $\mathbf{3 0}$, 60,90120 , and $250 \mathrm{msec}$, followed by a mask and $250 \mathrm{msec}$ without a mask. After all blocks had been administered once for each task, the procedure was repeated, so that each subject received 12 trials at each duration under each task, for a total of 216 trials. The order of tasks was counterbalanced across the subjects in each age group, and the order of durations within tasks was randomized for each subject separately. Each task was introduced by two practice trials and each block by cycling a practice card five times at the duration for that block. The sequence of each test trial was the fixation field presented for $750 \mathrm{msec}$, a dark interval of $100 \mathrm{msec}$, the stimulus array for the appropriate duration, and, immediately following the offset of the stimulus array, the masking stimulus for 250 msec (on trials with the mask). As in the practice trials, test trials were self-paced, four responses were required on each trial, children were rewarded for correct responses, and adults were given verbal feedback.

Children were given seven sessions: one practice session and two test sessions under each of the three tasks, such that each session was a different task. Adults were given two sessions, receiving practice trials and the first series of trials under each of the three tasks in the first session and the second series of trials under each task in the second session.

\section{RESULTS}

The $P$ task was scored for the mean number of $\times s$ placed in the correct cells of the matrix at each duration, the $F$ task for the mean number of correct forms placed anywhere on the square, and the $P \times F$ task for both the mean number of cells correctly filled disregarding form correctness and the mean number of correct forms disregarding correct placements. The means were subjected to a 2 (Age) $\times$ 2 (Alone vs. Combined Task) $\times 2$ (Position vs. Form Information) $\times 6$ (Stimulus Duration) analysis of variance.' All main effects were significant [for Age, $F(1,8)=23.4, p<.01 ;$ for Task, $F(1,8)=12.8$, $\mathrm{p}<.01$; for Type of Information, $F(1,8)=413.6$, $\mathrm{p}<.001$; and for Duration, $\mathrm{F}(5,40)=70.3, \mathrm{p}<.001$ ]. In addition, significant two-way interactions were obtained between Age and Type of Information $[F(1,8)=43.5, p<.001]$, between Age and Duration $[F(5,40)=13.4, p<.001]$, between Task and Duration $[F(5,40)=5.9, p<.001]$, and between Type of Information and Duration $[F(5,40)=14.4$, $\mathrm{p}<.001]$. Finally, significant three-way interactions were found between Age, Type of Information, and Duration $[F(5,40)=4.8, p<.01]$ and between Task, Type of Information, and Duration $[F(5,40)=3.9$, $\mathrm{p}<.011$.

The mean position scores ( $P$ scores) for the $P$ and $P \times F$ tasks at each duration for each age group are given in Figure 2, and the mean form scores (F scores) for the $F$ and $P \times F$ tasks at each duration for each age group are given in Figure 3. Differences between the shapes of these functions were tested by a trend analysis. Since the stimulus duration intervals were unequal, Gaito's (1965) method for deriving orthogonal coefficients was used, and, in accordance with these procedures, only the linear and quadratic components were computed. The trend tests were performed on durations 30 to $250 \mathrm{msec}$, followed by the mask, with the 250-msec duration without the mask eliminated. There was a significant overall linear trend $[F(1,8)=201.6 \mathrm{p}<.001]$, and there were significant effects of Age, Task, and Type of Information on linearity $[F(1,8)=45.6, p<.001$; $F(1,8)=7.7, p<.05 ;$ and $F(1,8)=24.6, p<.01$, respectively]. In addition, there was a significant Age by Type of Information interaction effect on the linear trend $[F(1,8)=10.9, p<.05]$ and a significant Task by Type of Information effect on the linear trend $[F(1,8)=10.1, p<.05]$. An analysis of the Type of Information effect on linearity was performed for each age group. For adults only, there was a significant effect of Type of Information on the linear trend $[F(1,4)=31.6, p<.01]$, confirming the impression that the adult functions for position information on Figure 2 show a linear increase with increasing stimulus duration, whereas the adult functions for form information on Figure 3 do not. An analysis of the Type of Information effect on linearity was also performed separately for each task. The significant effect was limited to the single task $[F(1,4)=10.2, p<.05]$. Thus, with scores collapsed across age groups, position information showed a linear increase with increasing duration only for the single task. As Figure 2 illustrates, this effect appears to be due primarily to the children, but the Age by Task by Type of Information effect on the linear trend did not reach significance.

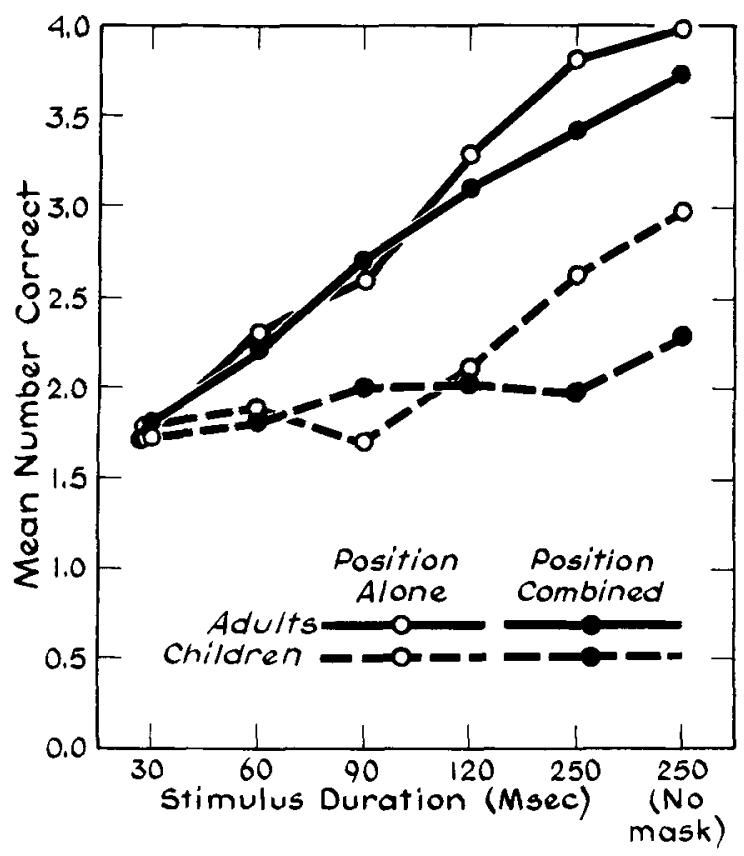

Figure 2. Mean position scores at each duration for the position information alone task ( $P$ task) and position plus form information task ( $P \times F$ task) for each age group. 


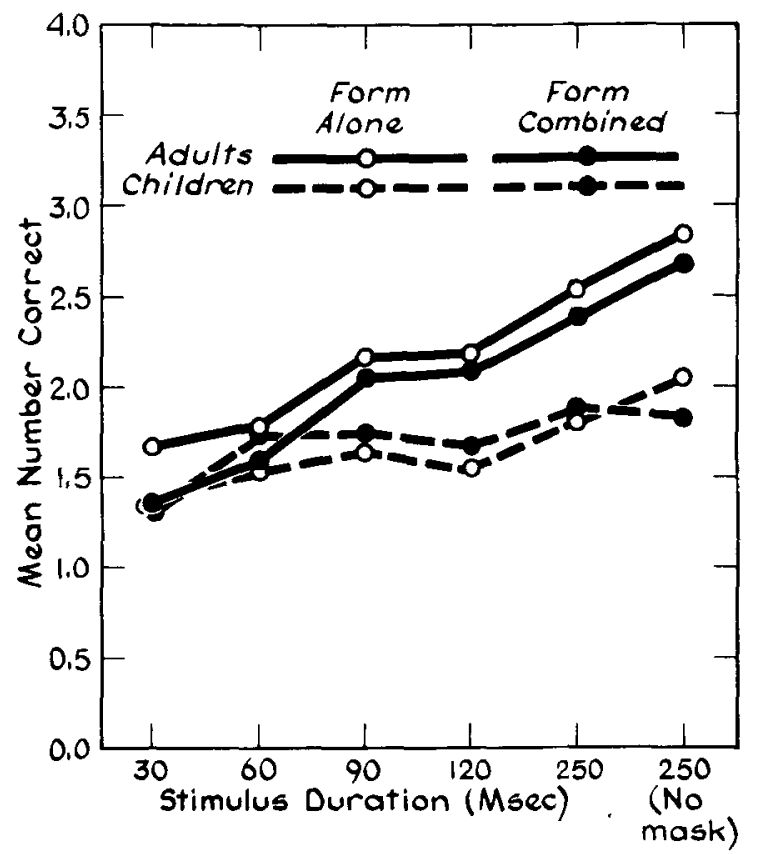

Figure 3. Mean form scores at each duration for the form information alone task ( $F$ task) and the position plus form information task (P $\times F$ task) for each age group.

There was also a significant overall quadratic trend $[F(1,8)=13.5, p<.01]$ and a significant effect of Age on the quadratic trend $[F(1,8)=6.2$, $\mathrm{p}<.05]$. An analysis of the quadratic trend performed separately for each age group revealed that it was limited to adults $[F(1,4)=14.3, p<.05]$.

\section{DISCUSSION}

The linear functions for adults on position information in this study confirm the previous findings that adults encode position information faster than identity information (Smythe \& Finkel, 1974; den Heyer, Note 1). With scores combined across tasks, children did not show a linear function for position information. However, their function for position information in the single task does show a linear increase after $90 \mathrm{msec}$ duration, contributing to the significant Task by Type of Information effect on linearity. This significant linear trend of position information in the single task reflects a loss of parity in position information across tasks. However, it is clear from Figure 2 that the loss of parity for position information occurs only for children. Form information, on the other hand, showed no loss of parity across tasks for either age group; it was not processed faster when only form information was presented and reported.

Thus, children, in contrast to adults, can encode position information faster when that is the only kind of information that they have to process and then only at longer durations. It is possible that the increase in their processing of position information alone after $90 \mathrm{msec}$ is due to being able to see the four filled cells as a pattern at the longer durations. Thus, at these durations, their strategy may shift to wholistic processing of the four filled cells as a gestalt. Adults are apparently still able to use this strategy when form information must also be reported, since their functions for position information in both the single and combined tasks are linear. Thus, they must be able to focus on the pattern of filled cells independently of the form information contained therein. For children, however, the presence of form information interferes with the processing of the four positions as a pattern. Their $P$ scores in the combined task do not differ appreciably from their $F$ scores in the single or combined tasks. Thus, unlike adults, they are unable to process these two types of information independently.

The loss of parity for position information only at the longest durations in this study agrees with the results of Smythe and Finkel (1974), who also found a loss of parity only for position information at mask delays of $90 \mathrm{msec}$ and longer, with no loss in form information. den Heyer (Note 1), however, found no loss of parity in position information, only a higher intercept by comparison with form information. Since den Heyer used eight position markers, while the other studies used only four, the absence of a differential gain in the position-alone task in his study may be due to an inability to process eight positions in a wholistic fashion. He attributes the difference found in intercept to a faster initiation of encoding or faster construction of the image for position information. Why the intercept difference was not found in the other studies is not easily explained.

It might be argued that the faster rate of processing position information in the position-alone task is due to differences in the masks used, the mask of $\times \mathrm{s}$ being less effective than the mask which combined parts of the forms. However, if the masking stimuli were differentially effective as stop-processing masks, then this difference should have been reflected at the shorter rather than the longer durations. It seems clear that position information is simply easier to process, but that for children, this is true only in the position-alone task. Adult $\mathbf{P}$ scores reached ceiling in the position-alone task and almost reached it in the combined task. In contrast, their $F$ scores did not quite attain a level of 3 out of 4 correct in either task. Children reached a level of 3 out of 4 positions correct on the position-alone task but only about 2 out of 4 forms correct on the single and combined tasks and little more than 2 out of 4 positions correct on the combined task. The two-item limit on form information is consistent with previous findings on STM capacity for forms in young children (Blake, 1974; Haith, Morrison, Sheingold, \& Mindes, 1970). 
The limit on $P$ scores in the position-alone task is lower than that found by Finkel (1973) but may be due to differences in scoring. In any case, for both groups, position information is encoded faster, perhaps because it contains less structure and is processed at a lower level in the system (Dick \& Dick, 1969; Neisser, 1967).

In conclusion, adults show no loss of parity in processing either position or form information when both types of information must be processed compared with processing only one type of information. Thus, they appear to process these two types of information in a parallel independent fashion. Children, on the other hand, show some loss of parity in their processing of position information when they must also process form information. Thus, their inability to process multiple-form arrays in a parallel independent fashion (Blake, 1974) is replicated in an inability to process two different kinds of information using a parallel independent strategy.

\section{REFERENCE NOTES}

1. den Heyer. K. Parallel encoding of alphabetic and twodimensional position information under conditions of backward masking. Paper presented at the meeting of the Canadian Psychological Association. Montreal. June 1972.

\section{REFERENCES}

BlaKe. J. Developmental change in visual information processing under backward masking. Journal of Experimental Child Psychology. 1974, 17, 133-146.

Cumming. G.. \& Coltheart, M. Short-term memory: Are item and position information stored independently? Psychonomic Science, 1969, 15, 216-218.

Dick, A. O. Relations between the sinsory register and short-term storage in tachistoscopic recognition. Joumal of Experimental Psychology, 1969, 82, 279-284.

Dick, A. O., \& Dick, S. O. An analysis of hierarchical processing in visual perception. Canadian Journal of Psychology, 1969, 23, 203-211.

FinKEL, D. L. A developmental comparison of the processing of two types of visual information. Joumal of Experimental Child Psychology, 1973, 16, 250-266.

FinKeL, D. L., \& SMYThe, L. Short-term storage of spatial information. Developmental Psychology, 1973, 9, 424-428.

Gairo, J. Unequal intervals and unequal $n$ in trend analyses. Psychological Bulletin, 1965, 63, 125-127.

Garner, W. R., \& Morton, J. Perceptual independence: Definitions, models, and experimental paradigms. Psychological Bulletin, 1969, 72, 233-259.

Haber, R. N., \& Standing, L. Clarity and recognition of masked and degraded stimuli. Psychonomic Science, 1968, 13, 83-84.

Haith, M. M., Morrison, F. J., Sheingold, K., \& Mindes, P. Short-term memory for visual information in children and adults. Journal of Experimental Child Psychology, 1970, 9. 454-469.

Liss, P. Does backward masking by visual noise stop stimulus processing? Perception \& Psychophysics, 1968, 4, 328-330.

NeIsser, U. Cognitive psychology. New York: Appleton-CenturyCrofts, 1967.

SMYTHE, L., \& FinKeL, D. L. Masking of spatial and identity information from geometric forms by a visual noise field. Canadian Journal of Psychology, 1974, 28, 399-407.

TURVEY, M. T..\& KRAVETz, S. Retrieval from iconic memory with shape as the selection criterion. Perception \& Psychophysics. $1970.8,171-172$.

\section{NOTE}

1. Blocks was not included as a factor because a previous analysis which compared scores on the $P$ and $F$ tasks with mean number of correct forms in correct position on the $P \times F$ task found only a main effect of Blocks with no interaction between Blocks and Age, Task, or Duration.

(Received for publication March 1, 1976; revision accepted August 3, 1976.) 